\title{
Effect of Gender and Various Diets on Bile Acid Profile and Related Genes in Mice ${ }^{\mathbb{S}}$
}

\author{
Chong Ma, (P) Ying Guo, and Curtis D. Klaassen
}

Department of Clinical Pharmacology, Xiangya Hospital, Central South University, Changsha, P. R. China (C.M., Y.G.); Institute of Clinical Pharmacology, Central South University, Hunan Key Laboratory of Pharmacogenetics, Changsha, P. R. China (C.M., Y.G.); Engineering Research Center of Applied Technology of Pharmacogenomics, Ministry of Education, Changsha, P. R. China (C.M., Y.G.); National Clinical Research Center for Geriatric Disorders, Xiangya Hospital, Central South University, Changsha, Hunan, P.R. China (C.M., Y.G.); and Department of Pharmacology, Toxicology and Therapeutics, University of Kansas Medical Center, Kansas City, Kansas (C.D.K.)

Received June 29, 2020; accepted October 2, 2020

\section{ABSTRACT}

Diet is an important factor for many diseases. Previous studies have demonstrated that several diets had remarkable effects on bile acid (BA) homeostasis, but no comprehensive information for both genders has been reported. Therefore, the current study characterized the nine most used laboratory animal diets fed to both genders of mice for a comparable evaluation of the topic. The results revealed that marked gender difference of BA homeostasis is ubiquitous in mice fed the various diets, and of the nine diets fed to mice, the atherogenic and calorie-restricted diets had the most marked effects on BA homeostasis, followed by the laboratory chow and essential fatty acid-deficient diets. More specifically, females had higher concentrations of total BAs in serum when fed six of the nine diets compared with male mice, and 26 of the 35 BA-related genes had marked gender difference in mice fed at least one diet. Although mice fed the calorie-restricted and atherogenic diets had increased BA, which was more pronounced in serum than liver, the intestinal farnesoid $X$ nuclear receptor-fibroblast growth factor 15 axis changed in the opposite direction and resulted in different hepatic expression patterns of Cyp7a1. Compared with AIN93M purified diet, higher hepatic expression of multidrug resistance-associated protein 3 was the only alteration in mice fed the laboratory chow diet. The other diets had little or no effect on BA concentrations in the liver and plasma or in the expression of BA-related genes. This study indicates that gender, the atherogenic diet, and the calorie-restricted diet have the most marked effects on BA homeostasis.

\section{SIGNIFICANCE STATEMENT}

Previous evidence suggested that various diets have effect on bile acid (BA) homeostasis; however, it is not possible to directly compare these findings, as they are all from different studies. The current study was the first to systematically investigate the influence of the nine most used experimental mouse diets on BA homeostasis and potential mechanism in both genders of mice and indicates that gender, the atherogenic diet, and the calorie-restricted diet have the most marked effects on BA homeostasis, which will aid future investigations.
This work was supported by the National Institutes of Health (Grants ES009649, ES-019487), the National Natural Scientific Foundation of China (Grant No. 81503563), Hunan Provincial Natural Science Foundation of China (2019JJ60074), and Hunan Provincial Innovation Foundation for Postgraduate (CX20190250).

https://doi.org/10.1124/dmd.120.000166.

S This article has supplemental material available at dmd.aspetjournals.org.

\section{Introduction}

Diets have important effects on organisms. Diets high in saturated fat and glucose, or with high ratios of n-6:n-3 polyunsaturated fatty acids, can cause disorders in body lipid, glucose, and energy metabolism, which may contribute to the development of metabolic syndrome (Berr et al., 1993; Vamecq et al., 1993; Bae et al., 2013; Luo and Yang, 2016; Luo et al., 2016; Bertaggia et al., 2017; Jiang et al., 2019; Liao et al., 2019). In contrast, a calorie-restricted diet fed to mice improves metabolic syndrome by altering the metabolism of bile acids (BAs) (De Guzman et al., 2013; Kok et al., 2018). BAs are important signaling

ABBREVIATIONS: ABC, ATP-binding cassette; ABCG5/8, ATP-binding cassette subfamily G members 5/8; ATP8B1, P-type ATPase, class 1, type 8B, member 1; ASBT, apical sodium-dependent bile salt transporter; BA, bile acid; BAAT, Bile acid-CoA: amino acid $N$-acyltransferase; BACS, Bile acyl-CoA synthetase; BCRP, Breast cancer resistance protein; BSEP, bile salt export pump; CA, cholic acid; CDCA, chenodeoxycholic acid; DCA, deoxycholic acid; EFA, essential fatty acid; FA, fatty acid; FGF15, fibroblast growth factor 15; FGFR4, fibroblast growth factor receptor 4; FXR, farnesoid X nuclear receptor; HDCA, hyodeoxycholic acid; LCA, lithocholic acid; LRH, Liver receptor homolog; LXR, Liver $x$ receptor; MCA, muricholic acid; MDCA, murideoxycholic acid; MDR, Multidrug resistance protein; MRP, multidrug resistance-associated protein; NTCP, sodium/ taurocholate cotransporting polypeptide; OATP, organic anion-transporting polypeptide; $12 \alpha-\mathrm{OH}, 12 \alpha$-hydroxylated; OST, organic solute transporter; PCA, principal component analysis; SHP, small heterodimer partner; STAR, Steroidogenic acute regulatory protein; TCA, taurocholic acid; TCDCA, taurochenodeoxycholic acid; TDCA, taurodeoxycholic acid; THDCA, taurohyodeoxycholic acid; TLCA, taurolithocholic acid; T $\omega \mathrm{MCA}$, tauro- $\omega \mathrm{MCA}$; TMDCA, tauromurideoxycholic acid; TUDCA, tauroursodeoxycholic acid; UDCA, ursodeoxycholic acid. 
molecules and metabolic regulators that can alter glucose and lipid homeostasis as well as insulin resistance in humans and mice (Makishima et al., 1999; Trauner et al., 2010; Tsuchida et al., 2012; Guo et al., 2016; Liu et al., 2018; Wang et al., 2019). Therefore, it is important to explore the effects of various diets on endogenous active substances such as BAs.

The primary BA chenodeoxycholic acid (CDCA) is synthesized from cholesterol in hepatocytes via two pathways: the classic pathway that utilizes cytochrome P450 7a1 (CYP7A1) and the alternative pathway that involves CYP7B1 (Chen et al., 2019) (see Fig. 1). CDCA is then converted to cholic acid (CA) via sterol $12 \alpha$-hydroxylase (CYP8B1) or $6 \beta$-hydroxylated and epimerized to $\alpha$-muricholic acid (MCA) and $\beta$ MCA in rodents by CYP2C70 (Takahashi et al., 2016; Donepudi et al., 2017). These primary BAs are conjugated predominantly with taurine in rodents and with glycine and taurine in humans by bile acyl-CoA synthetase and bile acid-CoA:amino acid $N$-acyltransferase and transferred to the intestine primarily via the bile salt export pump [BSEP/ATP-binding cassette (ABC) B11] (Xie et al., 2016; Droge et al., 2017).

Primary BAs are converted into secondary BAs by intestinal microbial enzymes with the oxidation and epimerization of the hydroxyl groups at $\mathrm{C} 3, \mathrm{C} 7$, and $\mathrm{C} 12$. Almost $95 \%$ of the BAs are reabsorbed from the intestine with the help of the apical sodium-dependent bile acid transporter (ASBT) and organic solute transporter (OST) $\alpha / \beta$. The portal vein transfers BAs to the liver where they are taken up by the sodium/ taurocholate cotransporting polypeptide (NTCP) and the organic aniontransporting polypeptide (OATP) 1b2. Although most of the BAs are excreted into bile by BSEP, a small amount of BAs is secreted by the liver back into the systemic circulation via $\operatorname{OST} \alpha / \beta$ and multidrug resistance-associated proteins (MRPs) 3 and 4.

BAs regulate their own homeostasis and perform other functions by binding to receptors in the intestine and liver. Farnesoid $\mathrm{X}$ nuclear receptor (FXR) is a ligand-activated transcription factor belonging to the nuclear receptor superfamily and is essential for regulating BA and lipid homeostasis. Activation of intestinal FXR suppresses the expression of CYP7A1 and CYP8B1, which inhibit BA synthesis in the liver (Goodwin et al., 2000; Holt et al., 2003). The primary BA, CDCA, activates FXR expression that can alter obesity and reverse insulin resistance caused by a high-fat diet (Thomas et al., 2008; Shihabudeen et al., 2015). The BA metabolizing pathway is shown in Fig. 1.

Scattered reports have demonstrated that diet has an effect on BA homeostasis in mice fed high-fat, Western, atherogenic, laboratory chow, AIN-93M purified, and calorie-restricted diets (Wanon et al., 1998; Phan et al., 2002; Fu and Klaassen, 2013; Dermadi et al., 2017; Ichimura et al., 2017), but some of these studies focused on the serum and/or liver BA profile or were only reported to influence total BAs, and it is not possible to directly compare these findings as they are all from different studies. Additionally, although gender differences in BA profiles have been reported in mice fed the normal laboratory, highfat, and Western diets, the results are inconsistent. For example, one study found that female mice had a higher hepatic concentration of BAs than males when fed a laboratory diet (Fu et al., 2012); however, another study found the opposite effect in mice fed the high-fat and Western diets (Jena et al., 2017; Xie et al., 2017). Therefore, gender diversity of BAs may be altered by diets; however, very limited comparable information has been reported on this topic. Thus, in the current study, nine frequently used experimental diets were given to male and female mice simultaneously for 3 weeks to assess early diet-induced changes on the concentration of 20 individual BAs in livers and serum and the relative mRNA expression of major BA-related genes in livers and ileum, which will provide systematic evidence for further investigation of dietary effects.

\section{Materials and Methods}

Ethics Statement. The animal housing facility at the University of Kansas Medical Center is accredited by the Association for Assessment and Accreditation of Laboratory Animal Care. All procedures were approved by the University of Kansas Medical Center's Institutional Animal Care and Use Committee.

Animals and Treatments. Seven-week-old male and female C57BL/6 mice were purchased from Charles River Laboratories, Inc. (Wilmington, MA), housed according to the American Animal Association Laboratory Animal Care guidance under a standard 12-hour dark-light cycle and humidity-controlled environment with a room temperature at approximately $25^{\circ} \mathrm{C}$, and acclimated for at least 1 week before treatment. Mice $(n=5$ per diet, gender $=$ male and female $)$ were divided into nine groups and fed one of the following diets, which were all purchased from Harlan Laboratories (Madison, WI). The diets included AIN-93M purified diet (TD.94048), high fructose diet (TD.89247), high-fat diet (TD.97070), Western diet (TD.88137), essential fatty acid (EFA)-deficient diet (TD.8422), low n-3 fatty acid (FA) diet (TD.00235 + 7\% sunflower oil), laboratory chow (TD.8604, natural ingredient diet), diet restriction (75\% of the diet TD.8604 consumed by ad lib feeding), and atherogenic diet (a modification of the Western diet plus $1.25 \%$ cholesterol and $0.5 \%$ cholic acid) for 3 weeks, which was described in detail previously (Renaud et al., 2014). Mice were euthanized in the morning (8:00-10:00 AM), and their blood and tissues were sampled as described in our previous study (Zhang and Klaassen, 2010).

BA Extraction and Quantification. Sample extraction and quantification of individual BAs by ultra-high-performance liquid chromatography-tandem mass spectrometry were performed according to methods described previously (Alnouti et al., 2008; Zhang and Klaassen, 2010). In this study, a total number of 20 BAs in serum and liver were detected by ultra-high-performance liquid chromatography-tandem mass spectrometry simultaneously. The standards included tauromurideoxycholic acid (TMDCA; kind gifts from Dr. Alan F. Hofmann, University of California, San Diego), CA, CDCA, deoxycholic acid (DCA), ursodeoxycholic acid (UDCA), lithocholic acid (LCA), taurochenodeoxycholic acid (TCDCA), taurodeoxycholic acid (TDCA), tauroursodeoxycholic acid (TUDCA), taurolithocholic acid (TLCA) (purchased from Sigma-Aldrich, St. Louis, MO), hyodeoxycholic acid (HDCA), murideoxycholic acid (MDCA), $\alpha$, $\beta, \omega \mathrm{MCA}$, taurocholic acid (TCA), taurohyodeoxycholic acid (THDCA), tauro (T) $\alpha$ and $\beta$ MCA (purchased from Steraloids, Inc., Newport, RI), and T $\omega$ MCA (synthesized according to previous methods; Zhang et al., 2012c), which were diluted with $50 \%$ methanol and spiked with internal standards ${ }^{2} \mathrm{H} 4-\mathrm{CDCA}$ to construct standard curves between 5 and 20,000 ng/ml. All standard curves were constructed using a $1 /$ concentration $^{2}$ weighted quadratic regression, and the correlation coefficient $\left(r^{2}\right)$ for all BAs was above 0.99 (Supplemental Table 1). The limit of detection (signal/noise ratio $=3$ ) for the various BAs was in the range of $5-10 \mathrm{ng} / \mathrm{ml}$, which equals $0.01-0.02 \mathrm{nmol} / \mathrm{ml}$.

RNA Extraction. RNA from liver and ileum was extracted using RNA-Bee reagent (Tel-Test, Inc., Friendswood, TX) according to the manufacturer's protocol. RNA concentrations were quantified using a NanoDrop1000 Spectrophotometer (NanoDrop Technologies, Wilmington, DE) at a wavelength of 260 $\mathrm{nm}$. RNA integrity was confirmed by agarose gel electrophoresis and ethidium bromide staining of $5 \mu \mathrm{g}$ of total RNA to visualize intact $18 \mathrm{~S}$ and $28 \mathrm{~S}$ bands.

Multiplexed Branched-DNA Assay. The multiplexed branched-DNA technique, a robust, high-throughput method used for characterizing mRNA expression, was used. Relative expression of genes in the livers of mice fed the various diets was quantified using the multiplexed branched-DNA assay (QuantiGene high volume branched DNA signal amplification kit; Panomics/Affymetrix), according to a previously published method (Cheng et al., 2005). The gene sequences were accessed from GenBank.

Quantification of Fxr, Osta, Ost $\beta$, Asbt, Fibroblast Growth Factor 15 mRNA Expression by Real-Time Quantitative Polymerase Chain Reaction Assay. The mRNA expression of $F x r, O s t \alpha, O s t \beta, A s b t$, and fibroblast growth factor $15(F g f 15)$ in the ileum of mice were quantified by real-time polymerase chain reaction assay as described previously (Renaud et al., 2014). Reverse transcription of RNA to cDNA was performed with the Applied Biosystems High Capacity Reverse Transcriptase kit (Applied Biosystems, Foster City, CA). All primers were synthesized by Integrated DNA Technologies (Coralville, IA).

Statistical Analysis. Data are expressed as means \pm S.D. $(n=4$ to 5$)$. Statistical analyses were performed with an IBM-SPSS 21.0 computer program (IBM, Armonk, NY) and SIMCA (v14; Umetrics, UmeÅ, Sweden). Two-way 


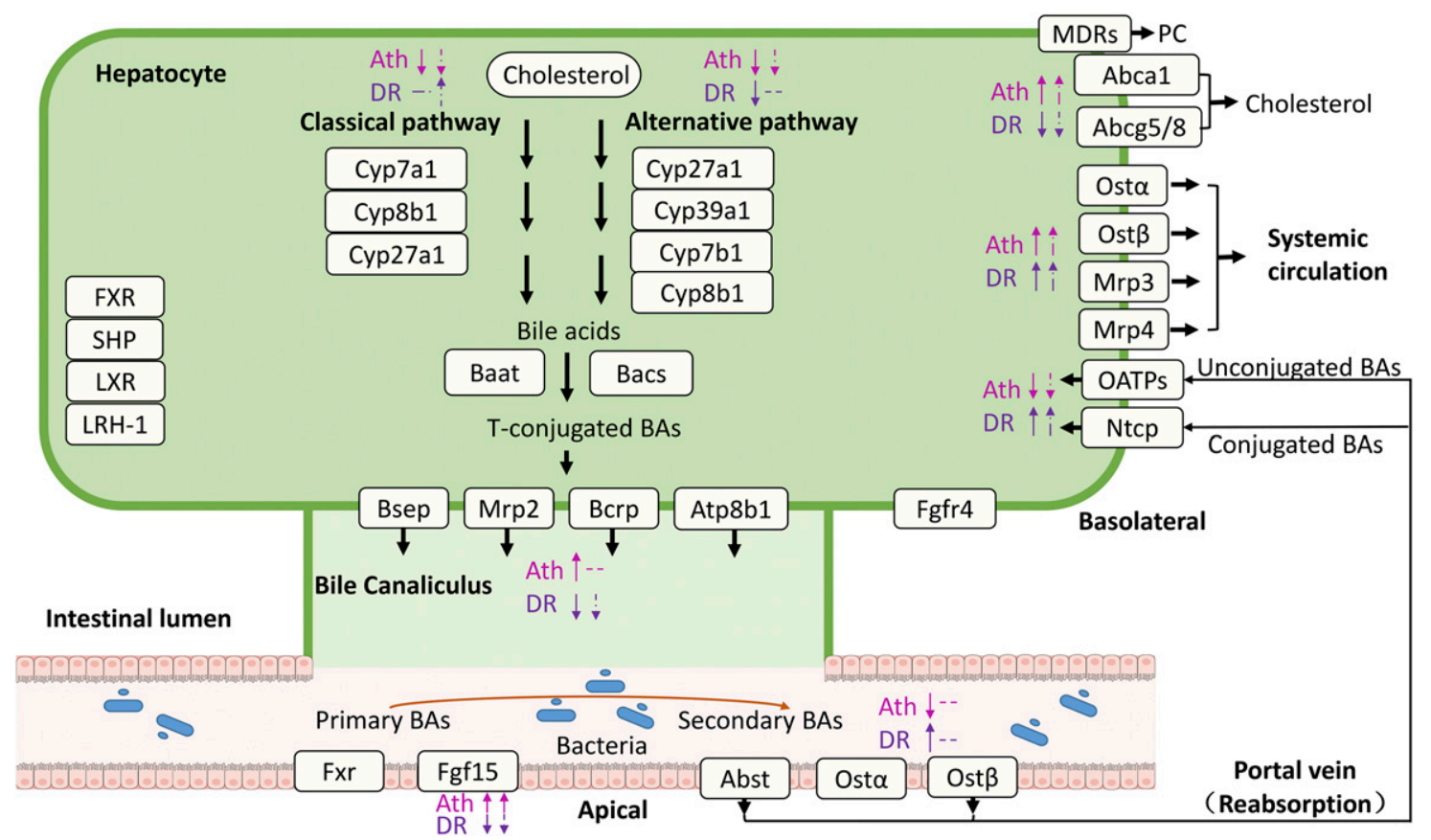


of male mice, and the right arrow (dashed line) shows the trend of female mice.

ANOVA followed by Duncan's post hoc test was used to analyze the differences between male and female mice fed the various diets. Principal component analysis (PCA) of the BAs and relative mRNA level of various diet treatments was determined by SIMCA. Statistical significance was set at $P<0.05$ for all analyses. AIN-93M purified diet is referred to as the control.

\section{Results}

\section{PCA Profile of BAs and Major BA-Related Genes in Livers of Mice Fed the Nine Diets}

To visualize patterns in the data and highlight similarities and differences between diets, PCA of the liver BAs and relative mRNA level of mice fed the various diets in both genders are shown in Fig. 2. Liver BAs and related mRNA profiles were substantially altered by the atherogenic and restricted diet compared with the AIN-93M purified diet (control) and the other diets. The profile of serum BAs had no significant change within mice fed the different diets in both genders (data not shown).

\section{Concentrations of BAs in Serum of Mice Fed the Nine Diets}

Gender. Ten taurine (T)-conjugated and 10 unconjugated BAs in serum were quantified in the current study, whereas only 17 BAs were detected (Supplemental Table 2). As shown in Fig. 3A, gender differences in total BAs, total T-conjugated BAs, and total unconjugated BAs in serum were observed in mice fed the various diets. The concentration of total BAs in the serum were higher in female than male mice fed six of the nine diets: the AIN-93M purified diet, the laboratory chow diet, the high fructose diet, the high-fat diet, the Western diet, and the low n-3 FA diet. Total T-conjugated BAs were also higher in the serum of female than male mice fed six of the nine diets: the AIN-93M purified diet, the laboratory chow diet, the high fructose diet, the high-fat diet, the low n-3 FA diet, and the EFA-deficient diet. Total unconjugated BAs were higher in the serum of female than male mice fed four of the nine diets: the AIN-93M purified diet, the high fructose diet, the Western diet, and the low n-3 FA diet.

As depicted in Fig. 3B, of the 17 individual BAs in the serum of mice fed the various diets in this study, 14 BAs show a significant gender difference in at least one diet. The concentrations of 14 BAs were higher in female than male mice fed the various diets, except that TCDCA was higher in male than female mice fed the atherogenic diet.

Female mice fed the AIN-93M purified diet had higher serum concentrations of primary BAs $\alpha \mathrm{MCA}, \beta \mathrm{MCA}$, UDCA, TCA, TCDCA, T $\alpha$ MCA, T $\beta$ MCA, and TUDCA as well as the secondary BAs DCA, HDCA, TDCA, T $\omega$ MCA, THDCA, and TMDCA than male mice. Female mice fed the high-fat diet had higher serum concentrations of BAs, including $\alpha \mathrm{MCA}, \beta \mathrm{MCA}$, UDCA, TCA, TCDCA, T $\alpha \mathrm{MCA}, \mathrm{T} \beta \mathrm{MCA}$, TUDCA, DCA, HDCA, TDCA, T $\omega$ MCA, THDCA, and TMDCA. Female mice fed the restricted diet had higher serum concentrations of TUDCA than male mice, whereas female mice fed the atherogenic diet also had higher concentrations of $\alpha \mathrm{MCA}, \beta \mathrm{MCA}, \omega \mathrm{MCA}$, and HDCA.

Diets. Some of the diets also affected BAs in serum. In male mice, the total BA concentrations in serum were increased in female mice by the atherogenic diet and the restricted diet, whereas the EFA-deficient diet decreased it (Fig. 3A). Meanwhile, total T-conjugated BAs were similarly increased in male mice fed the restricted diet and the atherogenic diet and were decreased in female mice by the EFAdeficient diet. However, total unconjugated BA concentrations in the serum of female mice did not change significantly with any diet, whereas in male mice, they were increased by the atherogenic diet.

Figure 3B indicates that 16 of the 17 individual BAs in serum of mice were affected by at least one diet. Moreover, female mice had higher serum BAs than male mice fed the various diets compared with the AIN93M purified diet. Specifically, the restricted diet significantly increased the serum concentration of primary BAs in male mice-CA, TCA, UDCA, $\alpha \mathrm{MCA}$, T $\alpha \mathrm{MCA}$, and T $\beta \mathrm{MCA}$ - as well as the secondary BAs DCA, TDCA, TMDCA, HDCA, THDCA, $\omega \mathrm{MCA}$, and T $\omega \mathrm{MCA}$. Whereas in female mice, the atherogenic diet significantly decreased the concentrations of TCDCA, T $\alpha$ MCA, T $\beta$ MCA, TUDCA, T $\omega$ MCA, TMDCA, and THDCA, in male mice, it increased the serum concentrations of CA, TCA, T $\alpha$ MCA, DCA, and TDCA and decreased the concentration of TUDCA.

The diets, other than the calorie-restricted diet and the atherogenic diet, also decreased BAs in the serum of female mice (Fig. 3B). 


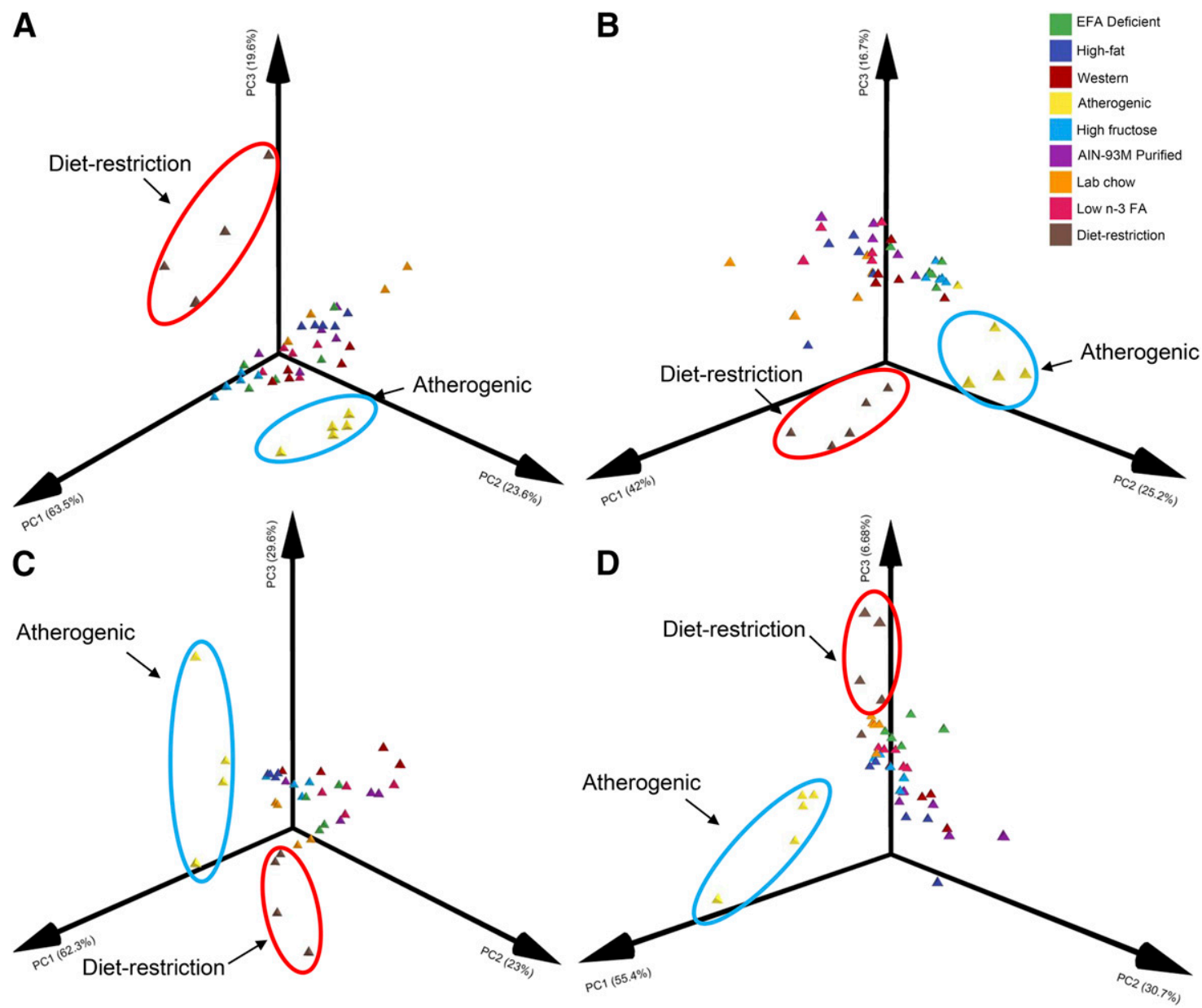

Fig. 2. Three-dimensional scatter plots of the PCA for the influence of different diets on liver BA profile and BA-related gene mRNA expression in both genders of mice. (A) PCA based on the BA-related gene mRNA expression in livers of male mice $(\mathrm{R} 2=0.797, \mathrm{Q} 2=0.532)$. (B) PCA based on the BA-related gene mRNA expression in livers of female mice $(\mathrm{R} 2=0.828, \mathrm{Q} 2=0.584)$. (C) PCA based on liver BA concentrations of male mice $(\mathrm{R} 2=0.988$, $\mathrm{Q} 2=0.664)$. (D) PCA based on liver BA concentrations of female mice $(\mathrm{R} 2=0.956, \mathrm{Q} 2=0.618)$. Each color represents a single diet. PCA was performed using the software SIMCA.

Specifically, the EFA-deficient diet decreased serum concentrations of T $\alpha$ MCA, TUDCA, TCA, TCDCA, and TDCA; the laboratory chow diet decreased $\beta$ MCA, TUDCA, DCA, and TDCA; the high fructose diet decreased T $\omega$ MCA, DCA, and TDCA; the Western diet decreased TCA and THDCA; and the high-fat diet and the low n-3 FA diet decreased the concentrations of DCA and TCA. In contrast to female mice, there were only two diets that had a significant influence on the concentrations of BAs in the serum of male mice. The laboratory chow diet increased the serum concentration of T $\omega \mathrm{MCA}$, and the EFA-deficient diet decreased TDCA.

\section{Concentrations of BAs in Livers of Mice Fed the Nine Diets}

Gender. Ten T-conjugated BAs and 10 unconjugated BAs were detected in livers (Supplemental Table 3). As shown in Fig. 4A, only a few gender differences in total BAs, total T-conjugated BAs, and total unconjugated BAs in livers were observed in mice fed the various diets. Specifically, a gender difference was detected in mice fed the highfat diet in which the total BAs in the livers of females was higher than in males, and the T-conjugated BAs were higher in the livers of females than males. In contrast, unconjugated BAs were higher in livers of male than in female mice fed the low n-3 FA diet and the EFA-deficient diet.

As is shown in Fig. 4B, of the 20 individual BAs in livers of male and female mice that were investigated in this study, 17 BAs show a significant gender difference after one or more of the diets. Specifically, female mice fed the AIN-93M purified diet had higher liver concentrations of the primary BAs CA, CDCA, UDCA, and TCA, as well as the secondary BAs DCA, MDCA, and TDCA than male mice, but the concentration of secondary BA T $\omega \mathrm{MCA}$ was higher in the livers of male mice. Male mice that were fed with the high fructose diet had a higher liver concentration of T $\omega$ MCA compared with female mice, but the concentration of CA was higher in the livers of female mice. However, mice fed the atherogenic diet, which produced a unique pattern of BAs in the livers compared with the other diets in both genders, did not show a significant gender difference of any BA.

Diets. Mice fed the various diets were compared with those fed the AIN-93M purified diet to determine the effect of the various diets on BA homeostasis. As is shown in Fig. 4A, total BAs and unconjugated BA concentrations in livers remained relatively constant in male mice fed the various diets, whereas in female mice, total BAs significantly decreased when fed the laboratory chow diet, and the unconjugated BAs were decreased by the calorie-restricted diet, the low n-3 FA diet, and the EFA-deficient diet. T-conjugated BAs did not change significantly in the livers of either gender of mice fed the nine diets.

Also shown in Fig. 4B, 19 of the 20 individual BAs in livers had gender differences in their response to at least one diet. Specifically, male mice fed the atherogenic diet had increased liver primary BAs CA, and TCA, but decreased $\beta \mathrm{MCA}, \mathrm{T} \beta \mathrm{MCA}$, and TUDCA. In the liver of 



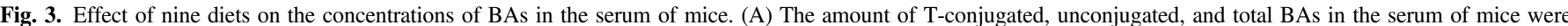



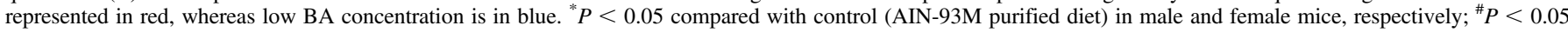
compared between genders fed the same diet.

male mice, the atherogenic diet increased secondary BAs DCA, and TDCA, but decreased $\omega \mathrm{MCA}, \mathrm{T} \omega \mathrm{MCA}, \mathrm{TMDCA}$, and THDCA. In comparison with the AIN-93M purified diet, female mice fed the atherogenic diet had decreased hepatic primary BAs CDCA, TCDCA, T $\alpha$ MCA, $\beta$ MCA, T $\beta$ MCA, UDCA, and TUDCA, and the secondary BAs $\omega$ MCA, T $\omega$ MCA, MDCA, TMDCA, and THDCA.
The concentration of BAs in the livers of male and female mice were also affected by the diets (Fig. 4B). Compared with the AIN-93M purified diet, the calorie-restricted diet increased liver concentrations of BAs in male mice including CA, TCA, DCA, and TDCA, the Western diet increased CDCA, and the high-fat diet decreased TLCA, T $\omega$ MCA, and THDCA. Meanwhile, in female mice, the calorie-restricted diet 


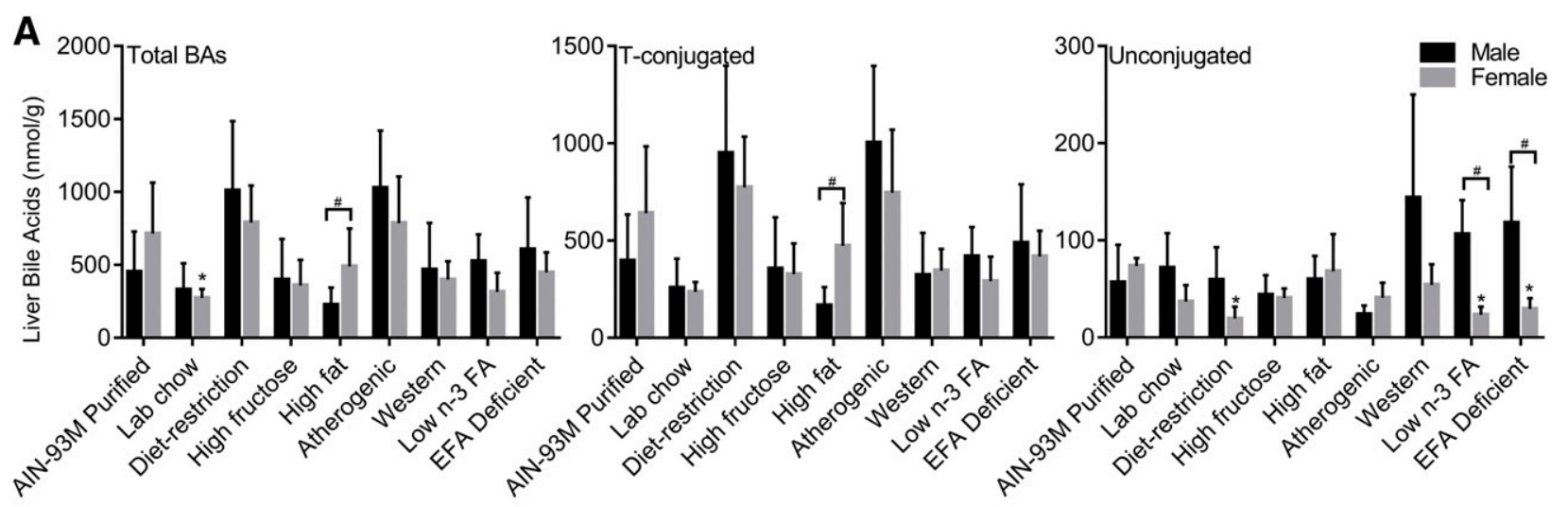

B



Group

Male

Female

Fig. 4. Effect of nine diets on the concentrations of BAs in the livers of mice. (A) The amount of T-conjugated, unconjugated, and total BAs in the livers of mice were quantified. (B) Heatmap of BAs in the livers of mice fed nine various diets. Average values of five replicates per diet are given by colored squares. High BA concentration is represented in red, whereas low BA concentration is in blue. ${ }^{*} P<0.05$ compared with control (AIN-93M purified diet) in male and female mice, respectively; ${ }^{\sharp} P<0.05$ compared between genders fed the same diet.

increased liver concentrations of T $\omega \mathrm{MCA}$, but decreased CDCA, $\alpha \mathrm{MCA}, \beta \mathrm{MCA}, \omega \mathrm{MCA}$, and MDCA. The high-fat diet decreased the liver concentrations of BAs in female mice including DCA, $\omega \mathrm{MCA}$, MDCA, THDCA, and TDCA; the low n-3 FA diet decreased CA, CDCA, $\alpha$ MCA, UDCA, DCA, and MDCA; the laboratory chow diet decreased the liver concentrations of T $\beta \mathrm{MCA}$, TUDCA, TDCA, and
TMDCA; the EFA-deficient diet decreased the liver concentrations of CDCA, UDCA, TDCA, and MDCA; the Western diet decreased the liver concentrations of CA, TCA, and THDCA; and the high fructose diet decreased the liver concentrations of DCA and $\omega \mathrm{MCA}$.

The Ratio of $12 \alpha$-Hydroxylated BAs to the Non-12 $\alpha$-Hydroxylated BAs. Compared with the AIN-93 purified diet, the atherogenic diet 
increased the ratio of $12 \alpha$-hydroxylated ( $12 \alpha-\mathrm{OH})$ BAs to non- $12 \alpha-\mathrm{OH}$ BAs in serum and livers of both genders (Table 1). The calorie-restricted diet also increased this ratio in livers of both genders and in the serum of male mice. The other six diets did not affect this ratio.

The mRNA Expression of BA-Synthetic Enzymes in Livers of Mice Fed the Nine Diets. Figure 5 and Supplemental Table 4 depict the effect of the nine diets on the mRNAs of the six BA-synthetic enzymes. The most pronounced gender difference was the expression of $C y p 7 b 1$, where female mice had little or no mRNA for this enzyme. This enzyme is a $7 \alpha$-hydroxylase that is responsible for the synthesis of 7-OH BAs in the alternative BA pathway (Chiang, 1998). This suggests that female mice produce little or no BAs by the alternative pathway. In contrast, the gene Cyp39al had higher expression in female mice when fed the AIN93M purified diet, the calorie-restricted diet, the high-fat diet, and the low n-3 FA diet.

As shown in Fig. 5, the mRNA expression of Cyp7al was increased by the calorie-restricted diet in female mice but was decreased by the atherogenic diet in both genders. Cyp $8 b 1$ was increased by the laboratory chow diet in male mice but was decreased by the calorierestricted diet and the atherogenic diet in both male and female mice. Cyp 27al was decreased by the high fructose diet and the EFA-deficient diet in female mice. Cyp $7 b 1$ in male mice was increased by the laboratory chow diet but was decreased by the restricted and the atherogenic diet. Cyp39al was only increased by the calorie-restricted diet in both genders. Bile acid-CoA:amino acid $\mathrm{N}$-acyltransferase was decreased by the calorie-restricted diet and the high fructose diet in male mice.

The mRNA Expression of BA-Transporters in Livers of Mice Fed the Nine Diets. Figure 5 and Supplemental Table 4 depict the effect of the nine diets on the mRNA expression of 12 transporters in livers. The most pronounced gender difference was the expression of breast cancer resistance protein, in which female mice had much less mRNA compared with males (Cheng and Klaassen, 2009; Klaassen and Aleksunes, 2010). The other gene that has a gender difference in expression is Oatpla1, in which the expression is about 2.5-fold higher in male than in female mice fed the various diets (Gong et al., 2011; Zhang et al., 2011, 2012a,b, 2013).

The mRNA expression of Ntcp was decreased by the Western diet in male mice and by the atherogenic diet in both genders. Oatplal was decreased by the high fructose diet and the atherogenic diet in male mice and by the calorie-restricted diet in both genders. Bsep was decreased by the calorie-restricted diet in female mice and increased by the atherogenic diet in male mice. Breast cancer resistance protein was decreased by the calorie-restricted diet in male mice. P-type ATPase, class 1 , type $8 \mathrm{~B}$, member 1 was decreased by the high fructose diet in female mice and the calorie-restricted diet in both genders. Ost $\beta$ was increased by the calorie-restricted diet in female mice and the atherogenic diet in both genders. Mrp3 was increased by the high-fat diet in female mice and the laboratory chow and the calorie-restricted diet in both genders. Mrp4 was increased by the calorie-restricted and the atherogenic diet.

The mRNA Expression of Cholesterol and Phosphatidylcholine Transporters in Livers of Mice Fed the Nine Diets. Figure 5 and Supplemental Table 4 depict the effect of the nine diets on the mRNA expression of the six cholesterol and phosphatidylcholine transporters. The most pronounced gender difference was the expression of multidrug resistance protein $1 b$, in which male mice had almost 1-fold higher levels compared with females (Lu and Klaassen, 2008; Cui et al., 2009). Mitochondrial cholesterol transporter steroidogenic acute regulatory protein was expressed higher in female mice fed the AIN-93 purified diet, the calorie-restricted diet, and the low n-3 diet. The mRNA expression of $A b c g 5$ and $A b c g 8$ were increased by the atherogenic diet in both genders. Abcal was decreased by the high fructose diet and the EFA-deficient diet in female mice and by the calorie-restricted diet in both genders. Liver multidrug resistance protein 2 was not affected by any of the diets.

The mRNA Expression of BA Receptors in Livers of Mice Fed the Nine Diets. Figure 5 and Supplemental Table 4 depict the effect of the nine diets on the mRNAs of five important receptors in BA homeostasis in the liver. The most pronounced gender difference was the expression of fibroblast growth factor receptor 4 ( Fgfr 4 ), where female mice fed five of the nine diets had more mRNA for the receptor than males. This is the receptor for Fgfl5 in the liver, which represses transcription of $C y p 7 a 1$. Female mice fed the calorie-restricted and low n-3 diets had increased Fxr expression compared with males. Compared with the AIN-93 purified diet, male mice fed the atherogenic diet had decreased Fxr expression, and liver $\mathrm{X}$ receptor was decreased by the restricted diet. Additionally, small heterodimer partner (Shp) was increased by the atherogenic diet in both genders, Fgfr 4 was decreased in female mice by the calorie-restricted diet, liver receptor homolog-1 was decreased in female mice on the high fructose diet, and both Fgfr 4 and liver receptor homolog-1 were decreased in females by the EFAdeficient diet.

The mRNA Expression of Genes Involved in BA Homeostasis in Duodenum of Mice Fed the Nine Diets. As shown in Fig. 5 and Supplemental Table 4, the mRNA expression of five genes involved in BA homeostasis in the ileum of mice were quantified by real-time quantitative polymerase chain reaction assay, and three of the genes were affected by at least one diet. Asbt was increased by the calorierestricted diet but was decreased by the atherogenic diet and the Western diet in the ileum of male mice. Ost $\beta$ was decreased by the Western diet in male mice. Fgf15, which was higher in the ileum of female than male mice fed the AIN-93 purified diet, was increased by the atherogenic diet but was decreased by the laboratory chow diet and the restricted diet in both genders.

\section{Discussion}

Previous studies reported the influence of gender and diet on serum and/or liver BA profile, and fewer studies discussed the classic BA regulatory pathways Fxr-Fgf15 and Fxr-Shp after diet intervention. Therefore, the influence of the nine most used experimental mouse diets on BA homeostasis were investigated simultaneously in the present study in both genders of mice. These data provide information on the importance of gender and diets on BA homeostasis in mice.

In serum, females had higher concentrations of total BAs than males when fed six of the nine diets (Fig. 3A), which is consistent with previous reports for normal laboratory and high-fat diets (Fu et al., 2012; Sheng et al., 2017; Wankhade et al., 2018). Our study is the first to show that females have higher concentrations of total BAs in mice fed the AIN-93M purified, high fructose, Western, and low n-3 FA diets. This higher concentration of BAs in serum of female mice may due to increased BA synthesis (Fig. 5, Cyp7al and Cyp39al), BA reabsorption (Fig. 5, Ntcp and Oatp2b1), or decreased BA efflux (Fig. 5 and Supplemental Table 5, Bsep). In addition, FXR is a critical regulatory factor of BA homeostasis via feedback mechanisms (Sheng et al., 2017). When BAs activate FXR in the intestine, there is an increase in FGF15 secreted into the portal circulation to interact with FGFR4 in the liver and to downregulate BA synthesis and transport (Mencarelli and Fiorucci, 2010). In the present study, Fxr may also contribute to the gender differences of BA metabolism in the above five diets, except the laboratory chow diet, since the altered expression of at least one gene involved in the Fxr-Fgf15-Fgfr4 feedback axis was detected (Fig. 5). Our study revealed this effect in mice fed the AIN-93M purified, 
TABLE 1

The ratio of $12 \alpha-\mathrm{OH}$ BAs to non- $12 \alpha-\mathrm{OH}$ BAs in livers and serum of two genders

$12 \alpha-$-OH BAs: CA, DCA, TCA, TDCA; non-12 $\alpha$-OH BAs: CDCA, LCA, UDCA, HDCA, MDCA, TCDCA, TLCA, TUDCA, TMDCA, THDCA, MCAs and TMCAs.

\begin{tabular}{|c|c|c|c|c|}
\hline \multirow{2}{*}{ Diet } & \multicolumn{2}{|c|}{ Male } & \multicolumn{2}{|c|}{ Female } \\
\hline & Liver & Serum & Liver & Serum \\
\hline AIN-93M purified & $0.24 \pm 0.05$ & $0.53 \pm 0.15$ & $0.57 \pm 0.05$ & $1.55 \pm 0.2$ \\
\hline Laboratory chow & $0.61 \pm 0.1$ & $0.69 \pm 0.15$ & $0.8 \pm 0.1$ & $1.28 \pm 0.25$ \\
\hline Diet-restriction & $2.12 \pm 0.55^{*}$ & $2.14 \pm 0.6^{*}$ & $1.65 \pm 0.15^{*}$ & $2.02 \pm 0.35$ \\
\hline High fructose & $0.47 \pm 0.45$ & $0.33 \pm 0.15$ & $0.8 \pm 0.05$ & $1.5 \pm 0.15$ \\
\hline High fat & $0.29 \pm 0.05$ & $0.5 \pm 0.20$ & $0.8 \pm 0.25$ & $1.51 \pm 0.35$ \\
\hline Atherogenic & $30.09 \pm 3.25^{*}$ & $48.47 \pm 9.8^{*}$ & $21.73 \pm 8.75^{*}$ & $41.24 \pm 16.35^{*}$ \\
\hline Western & $0.26 \pm 0.1$ & $0.61 \pm 0.2$ & $0.3 \pm 0.05$ & $0.82 \pm 0.2$ \\
\hline Low n-3 FA & $0.23 \pm 0.05$ & $0.56 \pm 0.25$ & $0.51 \pm 0.2$ & $1.53 \pm 0.35$ \\
\hline EFA deficient & $0.28 \pm 0.1$ & $0.22 \pm 0.1$ & $0.56 \pm 0.15$ & $1.05 \pm 0.3$ \\
\hline
\end{tabular}

"Indicates $P<0.05$ when compared with the AIN-93M purified diet. Mean values \pm S.D. are shown.

restricted, high-fat, atherogenic, and Western diets for the first time. This feedback mechanism may contribute to inhibit the global increase of serum BA concentration, and it is possible that the activation of this feedback axis may require longer time in mice fed the laboratory chow diet (Fu et al., 2012).

In liver, no obvious gender difference is first demonstrated for mice fed the AIN-93M purified, restricted, atherogenic, high fructose, low n-3 FA, and EFA-deficient diets in this study and is consistent with earlier report for the Western diet (Sheng et al., 2017). Previous studies have reported higher hepatic total BAs in female mice fed the laboratory chow and in male mice fed the high-fat diet; however, these effects were not observed in the current study, which may be due to the differences in the amount of time the mice were fed the diets and/or the type of experimental animal model used (Fu et al., 2012; Xie et al., 2017). In addition, although no gender difference of $F x r$ was found in livers of mice fed the high-fat diet, its target gene $S h p$, which has been reported to represses BA biosynthesis (Goodwin et al., 2000), was higher in female mice fed the high-fat diet. Thus, the hepatic Fxr-Shp feedback mechanism may be involved in the gender difference for mice fed the high-fat diet.

BAs are often quantified in serum and it is often assumed that BA concentrations in the serum reflects BA concentrations in the liver (Jiao et al., 2018). In the present study, BAs in the serum did not predict BA concentrations in the livers of mice. For example, the serum concentrations of $\alpha \mathrm{MCA}$, UDCA, HDCA, $\omega \mathrm{MCA}, \mathrm{T} \alpha \mathrm{MCA}$, and TMDCA were increased in male mice fed the calorie-restricted diet, whereas the hepatic concentrations of all these BAs remained stable (Figs. 3 and 4). Meanwhile, a review of the published data from a number of previous studies in mice also found a lack of correlation of changes in serum and liver BA concentrations in same and different mouse models as well as varied pathologic situations. For example, in the same $\mathrm{C} 57 \mathrm{BL} / 6 \mathrm{~J}$ mice, the serum concentrations of $\mathrm{CA}, \mathrm{CDCA}, \alpha \mathrm{MCA}$, and $\beta \mathrm{MCA}$ were increased in mice fed $30 \mathrm{mg} / \mathrm{kg}$ berberine compared with the control mice, whereas the liver concentrations of all of these BAs were not altered by berberine (Guo et al., 2016), and the same phenomenon was found in different mice models (germ-free and hepatocyte-specific

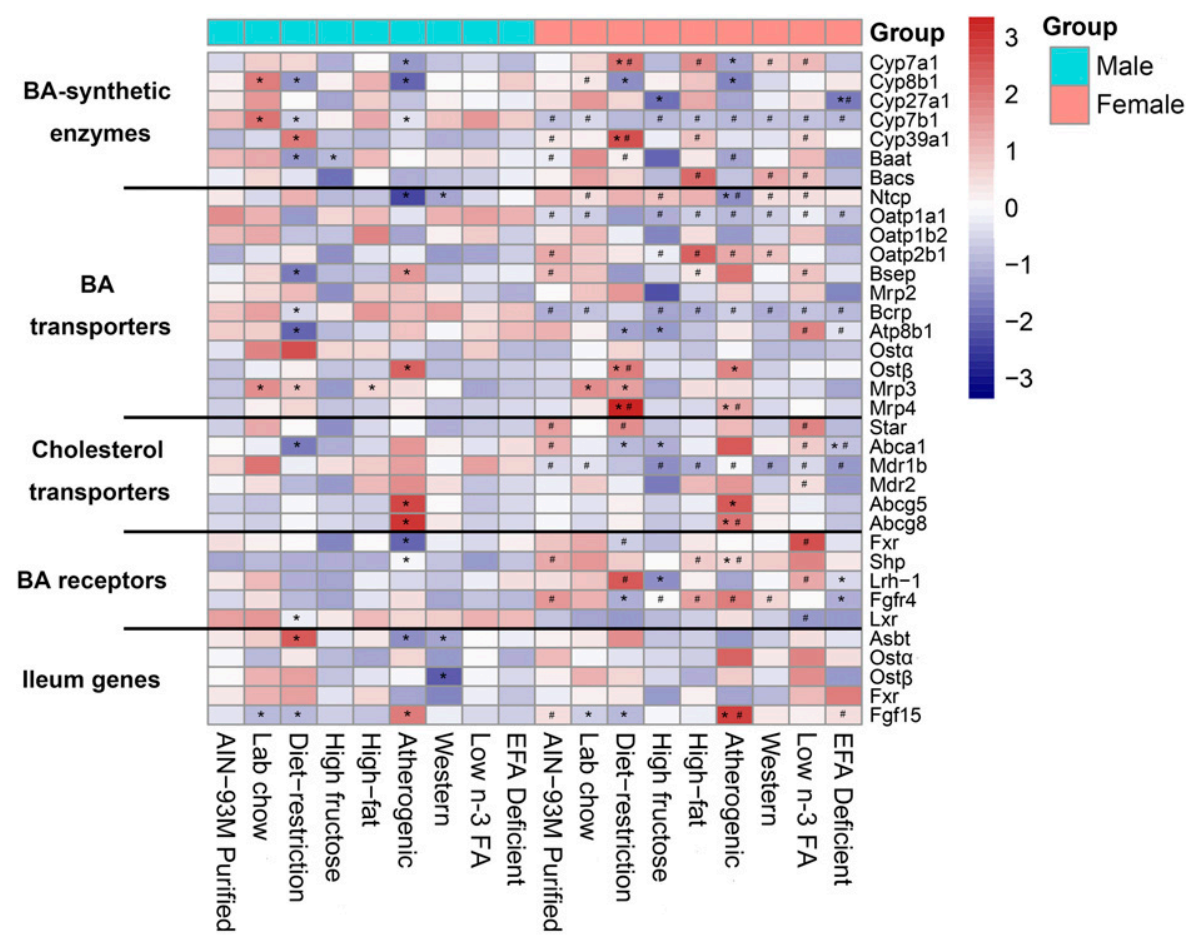

Fig. 5. Effect of nine diets on mRNA expression of BA-related genes in the livers and ileum of mice. Heatmap of BA-related genes in the livers and ileum of mice fed nine various diets. Average values of five replicates per diet are given by colored squares. High mRNA abundance is represented in red, whereas low mRNA abundance is in blue. Relative mRNA levels were calculated with male controls (male, AIN-93M purified diet) set as $100 \%$. Data were analyzed by twoway ANOVA. ${ }^{*} P<0.05$ compared with control (AIN$93 \mathrm{M}$ purified diet) in male and female mice, respectively; ${ }^{\#} P<0.05$ when data from the two genders fed the same diet are compared. 
deletion of NADPH cytochrome $\mathrm{P} 450$ reductase null mice) or in varied pathologic situations (mice after bile duct ligation) (Zhang et al., 2012b; Cheng et al., 2014; Selwyn et al., 2015). Thus, it is apparent from these numerous studies that changes in concentrations of BAs in the serum do not predict what happens to BA concentrations in the liver.

Of the nine diets fed to mice in the present study, the atherogenic and calorie-restricted diets had the largest effects on BA homeostasis. The atherogenic diet increased numerous BAs in the serum and liver, and the increase of total BAs in serum was more pronounced than that in the liver, which are novel findings. Meanwhile, the intestinal Fxr-Fgf15 negative feedback mechanism was apparent in mice fed the atherogenic diet, as there was a marked increase of Fgfl5 in the intestine (Fig. 5), and resulted in a decrease in BA synthetase (Cyp7al and Cyp8b1) (Fig. 5), which was consistent with a previous report (Gutierrez et al., 2006). In addition, Abcg5/Abcg8 are Fxr target genes and transporters that efflux cholesterol into the lumen of the intestine, and they were markedly increased in mice fed the atherogenic diet (Fig. 5), which was consistent with previous reports of CA-fed mice (Cui et al., 2009; Song et al., 2015). The addition of CA increased Abcg5/Abcg8 expression, which might inhibit the accumulation of cholesterol caused by the atherogenic diet, thereby delaying the occurrence of high cholesterol-related chronic diseases.

The atherogenic diet also increased the concentration of the $12 \alpha$ hydroxylated BAs CA and DCA and altered the ratio of $12 \alpha-\mathrm{OH}$ to non-12 $\alpha$-OH BAs in livers and serum of both genders of mice (Table 1). However, mice displayed a decreased expression of Cyp $8 b 1$, which is a sterol $12 \alpha$-hydroxylase and Fxr-Fgf15-Fgfr4 target gene. A previous study reported that the addition of $0.5 \% \mathrm{CA}$ to the diet suppresses the expression of Cyp8b1 in the livers of mice (Murphy et al., 2005). In addition, the increase of hepatic Shp might also be a potential regulator for this feedback inhibition of Cyp $8 b 1$ in mice fed the atherogenic diet (Yang et al., 2014).

The calorie-restricted diet did not have a consistent effect on the BA concentrations in serum or liver in the two genders. However, the calorie-restricted diet decreased Fgf15 in the intestine and decreased Cyp7b1; Cyp8b1; P-type ATPase, class 1, type 8B, member 1; and Abcal as well as increased Cyp39al and Mrp3 in the livers of both genders of mice. In addition, as discussed above, the serum BA concentration of female were higher than male mice, the present study found that the calorie-restricted diet closes the gap of the gender difference in serum by increasing BA concentration in male mice (total, T-conjugated BA, CA, TCA, DCA, TDCA). In addition, the calorierestricted diet increased the concentration of $12 \alpha-\mathrm{OH}$ BAs CA and DCA and the ratio of $12 \alpha-\mathrm{OH}$ to the non- $12 \alpha-\mathrm{OH}$ BAs in livers and serum of male mice (Table 1) and induced the feedback mechanism of Fxr-Fgf15 in the intestine, which resulted in a decrease of Cyp $8 \mathrm{bl}$.

As for the influence of diet, this is the first study to report the effect of high fructose, low n-3 FA, and EFA-deficient diets on the serum and hepatic BA profile in mice. Compared with the AIN-93M purified diet, there are little or no effects of these diets on BA homeostasis and related genes. This is also the first study to report that the only difference between mice fed the laboratory chow versus AIN-93M purified diet was that hepatic Mrp3 was higher in mice fed the laboratory chow diet. Previous studies in mice fed a high-fat or EFA-deficient diet reported that these diets inhibit the intestinal Fxr-Fgf15 pathway (Lukovac et al., 2009; Li et al., 2020); however, these results were not repeated in the current study. This discrepancy could be due to a relatively short duration of time on the diet. Therefore, the effects of the length of time on various diets should be considered in future studies to provide comprehensive insight into BA homeostasis.

In summary, the present study systematically investigated the influence of the nine most used experimental mouse diets on BA homeostasis in male and female mice. Several new findings were made: 1) marked gender differences in BA homeostasis is ubiquitous in mice fed various diets; 2) female mice had higher total BAs in serum than male mice fed the AIN-93M purified, high fructose, and low n-3 FA diets; 3 ) of the nine diets used, the atherogenic and calorie-restricted diets had the largest effects on BA homeostasis, followed by the laboratory chow and EFA-deficient diets, whereas the other four diets had less or no influence on BA homeostasis; 4) in serum, females had higher concentrations of total BAs than male mice fed the AIN-93M purified, high fructose, Western, and low n-3 FA diet; 5) changes in the concentrations of BAs in the serum do not always predict BA concentrations in the liver; and 6) the calorie-restricted diet closes the gap of the gender difference in serum and hepatic BA homeostasis found in controlled AIN-93M purified diet-fed mice. In conclusion, this study reveals the important role of gender and diet on BA homeostasis and underlying mechanisms, which will aid future investigations.

\section{Acknowledgments}

The authors would like to thank all the graduate students, postdoctoral fellows, and Xiaohong Lei who were in Dr. Klaassen's laboratory for technical support of the experiments.

\section{Authorship Contributions}

Participated in research design: Guo, Klaassen.

Conducted experiments: Guo.

Performed data analysis: Ma.

Wrote or contributed to the writing of the manuscript: Ma, Guo, Klaassen.

\section{References}

Alnouti Y, Csanaky IL, and Klaassen CD (2008) Quantitative-profiling of bile acids and their conjugates in mouse liver, bile, plasma, and urine using LC-MS/MS. J Chromatogr B Analyt Technol Biomed Life Sci 873:209-217.

Bae JS, Oh AR, and Cha JY (2013) Regulation of cholesterol metabolism in liver: link to NAFLD and impact of n-3 PUFAs. J Lifestyle Med 3:19-25.

Berr F, Goetz A, Schreiber E, and Paumgartner G (1993) Effect of dietary n-3 versus n-6 polyunsaturated fatty acids on hepatic excretion of cholesterol in the hamster. J Lipid Res 34: $1275-1284$.

Bertaggia E, Jensen KK, Castro-Perez J, Xu Y, Di Paolo G, Chan RB, Wang L, and Haeusler RA (2017) Cyp8b1 ablation prevents Western diet-induced weight gain and hepatic steatosis because of impaired fat absorption. Am J Physiol Endocrinol Metab 313:E121-E133.

Chen G, Zhang Q, Ai C, Huang S, Zhang H, Guo X, Wang W, Hua W, Bi H, and Wang H (2019) Serum metabolic profile characteristics of offspring rats before and after birth caused by prenatal caffeine exposure. Toxicology 427:152302.

Cheng X and Klaassen CD (2009) Tissue distribution, ontogeny, and hormonal regulation of xenobiotic transporters in mouse kidneys. Drug Metab Dispos 37:2178-2185.

Cheng X, Maher J, Chen C, and Klaassen CD (2005) Tissue distribution and ontogeny of mouse organic anion transporting polypeptides (Oatps). Drug Metab Dispos 33:1062-1073.

Cheng X, Zhang Y, and Klaassen CD (2014) Decreased bile-acid synthesis in livers of hepatocyteconditional NADPH-cytochrome P450 reductase-null mice results in increased bile acids in serum. J Pharmacol Exp Ther 351:105-113.

Chiang JY (1998) Regulation of bile acid synthesis. Front Biosci 3:d176-d193.

Cui YJ, Cheng X, Weaver YM, and Klaassen CD (2009) Tissue distribution, gender-divergent expression, ontogeny, and chemical induction of multidrug resistance transporter genes (Mdr1a, Mdr1b, Mdr2) in mice. Drug Metab Dispos 37:203-210.

De Guzman JM, Ku G, Fahey R, Youm YH, Kass I, Ingram DK, Dixit VD, and Kheterpal I (2013) Chronic caloric restriction partially protects against age-related alteration in serum metabolome Age (Dordr) 35:1091-1104.

Dermadi D, Valo S, Ollila S, Soliymani R, Sipari N, Pussila M, Sarantaus L, Linden J, Baumann M, and Nyström M (2017) Western diet deregulates bile acid homeostasis, cell proliferation, and tumorigenesis in colon. Cancer Res 77:3352-3363.

Donepudi AC, Boehme S, Li F, and Chiang JY (2017) G-protein-coupled bile acid receptor plays a key role in bile acid metabolism and fasting-induced hepatic steatosis in mice. Hepatology $\mathbf{6 5}$ : 813-827.

Dröge C, Bonus M, Baumann U, Klindt C, Lainka E, Kathemann S, Brinkert F, Grabhorn E, Pfister ED, Wenning D, et al. (2017) Sequencing of FIC1, BSEP and MDR3 in a large cohort of patients with cholestasis revealed a high number of different genetic variants. $J$ Hepatol 67:1253-1264

Fu ZD, Csanaky IL, and Klaassen CD (2012) Gender-divergent profile of bile acid homeostasis during aging of mice. PLoS One 7:e32551.

Fu ZD and Klaassen CD (2013) Increased bile acids in enterohepatic circulation by short-term calorie restriction in male mice. Toxicol Appl Pharmacol 273:680-690.

Gong L, Aranibar N, Han YH, Zhang Y, Lecureux L, Bhaskaran V, Khandelwal P, Klaassen CD, and Lehman-McKeeman LD (2011) Characterization of organic anion-transporting polypeptide (Oatp) $1 \mathrm{a} 1$ and $1 \mathrm{a} 4$ null mice reveals altered transport function and urinary metabolomic profiles. Toxicol Sci 122:587-597.

Goodwin B, Jones SA, Price RR, Watson MA, McKee DD, Moore LB, Galardi C, Wilson JG, Lewis MC, Roth ME, et al. (2000) A regulatory cascade of the nuclear receptors FXR, SHP-1, and LRH-1 represses bile acid biosynthesis. Mol Cell 6:517-526. 
Guo Y, Zhang Y, Huang W, Selwyn FP, and Klaassen CD (2016) Dose-response effect of berberine on bile acid profile and gut microbiota in mice. BMC Complement Altern Med 16:394. Gutierrez A, Ratliff EP, Andres AM, Huang X, McKeehan WL, and Davis RA (2006) Bile acids decrease hepatic paraoxonase 1 expression and plasma high-density lipoprotein levels via FXRmediated signaling of FGFR4. Arterioscler Thromb Vasc Biol 26:301-306.

Holt JA, Luo G, Billin AN, Bisi J, McNeill YY, Kozarsky KF, Donahee M, Wang DY, Mansfield TA, Kliewer SA, et al. (2003) Definition of a novel growth factor-dependent signal cascade for the suppression of bile acid biosynthesis. Genes Dev 17:1581-1591.

Ichimura M, Masuzumi M, Kawase M, Sakaki M, Tamaru S, Nagata Y, Tanaka K, Suruga K, Tsuneyama K, Matsuda S, et al. (2017) A diet-induced Sprague-Dawley rat model of nonalcoholic steatohepatitis-related cirrhosis. J Nutr Biochem 40:62-69.

Jena PK, Sheng L, Liu HX, Kalanetra KM, Mirsoian A, Murphy WJ, French SW, Krishnan VV, Mills DA, and Wan YY (2017) Western diet-induced dysbiosis in farnesoid X receptor knockout mice causes persistent hepatic inflammation after antibiotic treatment. Am J Pathol 187: $1800-1813$.

Jiang M, Li C, Liu Q, Wang A, and Lei M (2019) Inhibiting ceramide synthesis attenuates hepatic steatosis and fibrosis in rats with non-alcoholic fatty liver disease. Front Endocrinol (Lausanne) 10:665.

Jiao N, Baker SS, Chapa-Rodriguez A, Liu W, Nugent CA, Tsompana M, Mastrandrea L, Buck MJ, Baker RD, Genco RJ, et al. (2018) Suppressed hepatic bile acid signalling despite elevated production of primary and secondary bile acids in NAFLD. Gut 67:1881-1891.

Klaassen CD and Aleksunes LM (2010) Xenobiotic, bile acid, and cholesterol transporters: function and regulation. Pharmacol Rev 62:1-96.

Kok DEG, Rusli F, van der Lugt B, Lute C, Laghi L, Salvioli S, Picone G, Franceschi C, Smidt H, Vervoort J, et al. (2018) Lifelong calorie restriction affects indicators of colonic health in aging C57Bl/6J mice. J Nutr Biochem 56:152-164

Li H, Xi Y, Xin X, Tian H, and Hu Y (2020) Salidroside improves high-fat diet-induced nonalcoholic steatohepatitis by regulating the gut microbiota-bile acid-farnesoid $\mathrm{X}$ receptor axis. Biomed Pharmacother 124:109915.

Liao J, Liu B, Zhong W, Wang GD, Xu YL, and Chen X (2019) Protective effect of Lycium barbarum polysaccharides against high-fat diet-induced renal injury and lipid deposition in rat kidneys. J Biol Regul Homeost Agents 33:7-17.

Liu T, Yang H, Fan W, Tu J, Li TWH, Wang J, Shen H, Yang J, Xiong T, Steggerda J, et al. (2018) Mechanisms of MAFG dysregulation in cholestatic liver injury and development of liver cancer. Gastroenterology 155:557-571.e14

Lu H and Klaassen C (2008) Gender differences in mRNA expression of ATP-binding cassette efflux and bile acid transporters in kidney, liver, and intestine of 5/6 nephrectomized rats. Drug Metab Dispos 36:16-23.

Lukovac S, Los EL, Stellaard F, Rings EHHM, and Verkade HJ (2009) Effects of essential fatty acid deficiency on enterohepatic circulation of bile salts in mice. Am J Physiol Gastrointest Liver Physiol 297:G520-G531.

Luo L and Yang T (2016) Triptolide inhibits the progression of atherosclerosis in apolipoprotein E/ mice. Exp Ther Med 12:2307-2313.

Luo T, Miranda-Garcia O, Adamson A, Hamilton-Reeves J, Sullivan DK, Kinchen JM, and Shay NF (2016) Consumption of walnuts in combination with other whole foods produces physiologic, metabolic, and gene expression changes in obese C57bl/6J high-fat-fed male mice. J Nutr 146: $1641-1650$.

Makishima M, Okamoto AY, Repa JJ, Tu H, Learned RM, Luk A, Hull MV, Lustig KD, Mangelsdorf DJ, and Shan B (1999) Identification of a nuclear receptor for bile acids. Science 284: 1362-1365.

Mencarelli A and Fiorucci S (2010) FXR an emerging therapeutic target for the treatment of atherosclerosis. J Cell Mol Med 14:79-92.

Murphy C, Parini P, Wang J, Björkhem I, Eggertsen G, and Gåfvels M (2005) Cholic acid as key regulator of cholesterol synthesis, intestinal absorption and hepatic storage in mice. Biochim Biophys Acta 1735:167-175.

Phan J, Pesaran T, Davis RC, and Reue K (2002) The Diet1 locus confers protection against hypercholesterolemia through enhanced bile acid metabolism. J Biol Chem 277:469-477.

Renaud HJ, Cui JY, Lu H, and Klaassen CD (2014) Effect of diet on expression of genes involved in lipid metabolism, oxidative stress, and inflammation in mouse liver-insights into mechanisms of hepatic steatosis. PLoS One 9:e88584.

Selwyn FP, Csanaky IL, Zhang Y, and Klaassen CD (2015) Importance of large intestine in regulating bile acids and glucagon-like peptide-1 in germ-free mice. Drug Metab Dispos 43 : $1544-1556$.

Sheng L, Jena PK, Liu HX, Kalanetra KM, Gonzalez FJ, French SW, Krishnan VV, Mills DA, and Wan YY (2017) Gender differences in bile acids and microbiota in relationship with gender dissimilarity in steatosis induced by diet and FXR inactivation. Sci Rep 7:1748.
Shihabudeen MS, Roy D, James J, and Thirumurugan K (2015) Chenodeoxycholic acid, an endogenous FXR ligand alters adipokines and reverses insulin resistance. Mol Cell Endocrinol 414 $19-28$.

Song P, Rockwell CE, Cui JY, and Klaassen CD (2015) Individual bile acids have differential effects on bile acid signaling in mice. Toxicol Appl Pharmacol 283:57-64.

Takahashi S, Fukami T, Masuo Y, Brocker CN, Xie C, Krausz KW, Wolf CR, Henderson CJ, and Gonzalez FJ (2016) Cyp2c70 is responsible for the species difference in bile acid metabolism between mice and humans. J Lipid Res 57:2130-2137.

Thomas C, Pellicciari R, Pruzanski M, Auwerx J, and Schoonjans K (2008) Targeting bile-acid signalling for metabolic diseases. Nat Rev Drug Discov 7:678-693

Trauner M, Claudel T, Fickert P, Moustafa T, and Wagner M (2010) Bile acids as regulators of hepatic lipid and glucose metabolism. Dig Dis 28:220-224.

Tsuchida T, Shiraishi M, Ohta T, Sakai K, and Ishii S (2012) Ursodeoxycholic acid improves insulin sensitivity and hepatic steatosis by inducing the excretion of hepatic lipids in high-fat diet-fed KK-Ay mice. Metabolism 61:944-953.

Vamecq J, Vallee L, de la Porte PL, Fontaine M, de Craemer D, van den Branden C, Lafont H, Grataroli R, and Nalbone G (1993) Effect of various n-3/n-6 fatty acid ratio contents of high fat diets on rat liver and heart peroxisomal and mitochondrial beta-oxidation. Biochim Biophys Acta 1170: $151-156$

Wang C, Yang M, Zhao J, Li X, Xiao X, Zhang Y, Jin X, and Liao M (2019) Bile salt (glycochenodeoxycholate acid) induces cell survival and chemoresistance in hepatocellular carcinoma. J Cell Physiol 234:10899-10906.

Wankhade UD, Zhong Y, Kang P, Alfaro M, Chintapalli SV, Piccolo BD, Mercer KE, Andres A, Thakali KM, and Shankar K (2018) Maternal high-fat diet programs offspring liver steatosis in a sexually dimorphic manner in association with changes in gut microbial ecology in mice. $S c i$ Rep 8:16502

Wanon J, Guertin F, Brunet S, Delvin E, Gavino V, Bouthillier D, Lairon D, Yotov W, and Levy E (1998) The effects of cholesterol uptake from high-density lipoprotein subfractions on biliary sterol secretion in rats with essential fatty-acid deficiency. Hepatology 27:779-786.

Xie G, Wang X, Huang F, Zhao A, Chen W, Yan J, Zhang Y, Lei S, Ge K, Zheng X, et al. (2016) Dysregulated hepatic bile acids collaboratively promote liver carcinogenesis. Int J Cancer 139: $1764-1775$.

Xie G, Wang X, Zhao A, Yan J, Chen W, Jiang R, Ji J, Huang F, Zhang Y, Lei S, et al. (2017) Sexdependent effects on gut microbiota regulate hepatic carcinogenic outcomes. Sci Rep 7:45232.

Yang F, He Y, Liu HX, Tsuei J, Jiang X, Yang L, Wang ZT, and Wan YJ (2014) All-trans retinoic acid regulates hepatic bile acid homeostasis. Biochem Pharmacol 91:483-489.

Zhang Y, Csanaky IL, Cheng X, Lehman-McKeeman LD, and Klaassen CD (2012a) Organic anion transporting polypeptide 1a1 null mice are sensitive to cholestatic liver injury. Toxicol Sci 127: 451-462.

Zhang Y, Csanaky IL, Lehman-McKeeman LD, and Klaassen CD (2011) Loss of organic anion transporting polypeptide $1 \mathrm{a} 1$ increases deoxycholic acid absorption in mice by increasing in testinal permeability. Toxicol Sci 124:251-260.

Zhang Y, Csanaky IL, Selwyn FP, Lehman-McKeeman LD, and Klaassen CD (2013) Organic anion-transporting polypeptide 1a4 (Oatp1a4) is important for secondary bile acid metabolism. Biochem Pharmacol 86:437-445.

Zhang Y, Hong JY, Rockwell CE, Copple BL, Jaeschke H, and Klaassen CD (2012b) Effect of bile duct ligation on bile acid composition in mouse serum and liver. Liver Int 32:58-69.

Zhang Y and Klaassen CD (2010) Effects of feeding bile acids and a bile acid sequestrant on hepatic bile acid composition in mice. J Lipid Res 51:3230-3242.

Zhang Y, Limaye PB, Lehman-McKeeman LD, and Klaassen CD (2012c) Dysfunction of organic anion transporting polypeptide 1a1 alters intestinal bacteria and bile acid metabolism in mice. PLoS One 7:e34522.

Address correspondence to: Ying Guo, Department of Clinical Pharmacology, Xiangya Hospital, Institute of Clinical Pharmacology, Central South University, Hunan Key Laboratory of Pharmacogenetics, 110 Xiangya Rd., Changsha 410078, P. R. China; Engineering Research Center of Applied Technology of Pharmacogenomics, Ministry of Education, 110 Xiangya Rd., Changsha 410078, P. R. China. E-mail: guoying881212@csu.edu.cn; or Curtis D. Klaassen, University of Kansas Medical Center, Kansas City, KS 66160; 2617 W 112th St., Leawood, KS 66211. E-mail: curtisklaassenphd@gmail.com 